\title{
Thin, transparent conductive films fabricated from conducting polymer nanofibers
}

\author{
Borjigin Aronggaowa, Masahiro Kawasaki and Takeshi Shimomura
}

In this study, doped films consisting of a nanofiber network of a conducting polymer, poly(3-hexylthiophene), were fabricated, and optical transmittance and electrical conductivity measurements were carried out. It was found that the films could be doped more efficiently than the conventional non-nanofibrillar polymer films by dipping the films in a dopant solution. The conductivity of the nanofiber films was $34 \mathrm{~S} \mathrm{~cm},{ }^{-1}$ which was seven times larger than that of a conventional polymer film with the same transmittance $(\sim 85 \%)$. The superior transparency and conductivity properties of the nanofiber films were attributed to the existence of highly effective conducting pathways, a large void fraction generated by the bulky network of the nanofibers, and a high dopant concentration that results from the large surface area.

Polymer Journal (2013) 45, 819-823; doi:10.1038/pj.2012.214; published online 5 December 2012

Keywords: conducting polymer; nanofiber; organic electronics; poly(3-hexylthiophene)

\section{INTRODUCTION}

Materials that are both optically transparent and electrically conductive have attracted great interest because of their usefulness in applications such as solar cells, flat-panel displays, touch-sensitive screens, light-emitting diodes and photovoltaic cells. Indium tin oxide (ITO) has become the standard material for such applications. Commercially available ITO films with a thickness of $150-200 \mathrm{~nm}$ have a $>90 \%$ transmittance at visible wavelengths and a sheet resistance of less than $8 \Omega \mathrm{t}^{-1}$. However, the cost of indium is making ITO an increasingly expensive material, and because of its brittle nature, it is not suitable for newer applications, such as flexible devices.

As a substitute for ITO, films made from conducting polymers such as polyaniline composite, ${ }^{1-5}$ poly(3,4-ethylenedioxythiophene)/ poly(styrenesulfonate), ${ }^{6-10}$ and poly(3-alkylthiophene) ${ }^{11}$ have been investigated. These polymers have the advantage of being light in weight, highly flexible and chemically stable. However, conducting polymers typically exhibit absorption in the visible wavelength range and are thus considered unsuitable for applications that require high conductivity to be obtained in thicker films.

Alternatively, conducting polymer nanofibers of polyacetylene, ${ }^{12,13}$ polyaniline, ${ }^{14-16}$ and poly(3-alkylthiophene), ${ }^{17,18}$ with diameters in the range of tens of nanometers, have been reported in the last two decades. Using entangled networks of these nanofibers may overcome the transparency problems associated with conducting polymer films because the nanofibers do not easily aggregate or have the tendency to form bulky network structures. This nanofiber structure provides a combination of effective conducting pathways and a large number of voids without any absorbance. Compared with conventional nonnanofibrillar films, in which the polymer chains aggregate with a uniformly high density, films consisting of a bulky network of nanofibers can exhibit a much higher optical transmittance due to the large void fraction. A transparent conductive film consisting of a $1 \mu \mathrm{m}$-thick composite of polyaniline nanofibers and poly(methyl methacrylate) has been reported to exhibit a transmittance of $>40 \%$ in the visible region and a conductivity ranging from $10^{-4}$ to $10^{-2} \mathrm{~S} \mathrm{~cm}^{-1}{ }^{19}$

Recently, we investigated the field-effect transistor property of isolated regioregular poly(3-hexylthiophene) (rr-P3HT) nanofibers using fine electrodes with a spacing of $200 \mathrm{~nm}$. Mobilities of up to $5.6 \times 10^{-2} \mathrm{~cm}^{2} \mathrm{~V}^{-1} \mathrm{~s}^{-1}$ were measured; this value is higher than that observed for conventional P3HT films. ${ }^{20,21}$ Several groups have also reported an enhancement of carrier mobility for P3HT nanofibers. ${ }^{18,22-28}$ Thus, in the present study, doped films of P3HT nanofiber networks were fabricated, and transmittance and conductivity measurements were obtained. The results were compared with those for conventional P3HT films. In addition, because there have been almost no reports on the chemical stability of the doping state in P3HT nanofibers in air, time-dependent transmittance and conductivity measurements were performed to evaluate this doping stability.

\section{EXPERIMENTAL PROCEDURE}

rr-P3HT $\left(M_{w}=87000\right)$ was purchased from Aldrich Co. Inc. (St Louis, MO, USA) and used without further purification. Conducting polymer nanofibers were prepared using the following procedure. ${ }^{17} \mathrm{~A} 0.05 \%$ wt P3HT solution was prepared by adding rr-P3HT $(25 \mathrm{mg})$ to a flask containing a mixture of organic 
solvents $(50 \mathrm{~g})$, and stirring at a high temperature $\left(>70^{\circ} \mathrm{C}\right)$. The solvent mixture contained anisole as a poor solvent and chloroform as a good solvent at a ratio of $3: 7 \mathrm{v} / \mathrm{v}$, which was previously found to produce nanofibers with a high carrier mobility. ${ }^{21}$ After the reflux was stopped, the solution was cooled gradually from 70 to $20^{\circ} \mathrm{C}$ at a rate of $25^{\circ} \mathrm{Ch}^{-1}$, and the formation of nanofibers was indicated by a change in solution color from a transparent yellow to a turbid reddish brown. The nanofiber suspension was then spin-cast onto a glass-slide substrate for $1 \mathrm{~min}$ at 3000 r.p.m. The thickness of the resultant film was controlled by the number of drops of suspension used during the spin-casting process.

In comparison, a conventional non-nanofibrillar P3HT film was prepared using the following process. A $0.05 \%$ wt P3HT solution was prepared by adding rr-P3HT $(25 \mathrm{mg})$ to a flask containing only chloroform and stirring at a high temperature $\left(>50^{\circ} \mathrm{C}\right)$. After the reflux was stopped, the solution was cooled gradually from 50 to $20^{\circ} \mathrm{C}$ at a rate of $25^{\circ} \mathrm{Ch}^{-1}$, during which time the solution remained yellow. The P3HT solution was then spin-cast onto a glass-slide substrate using the same procedure as previously described for the nanofiber film.

Doping was performed by dipping the film-coated substrates for $1 \mathrm{~min}$ into an acceptor solution $(0.1 \mathrm{M})$, in which $\mathrm{FeCl}_{3}(81.0 \mathrm{mg})$ or $\mathrm{AuCl}_{3}(151.7 \mathrm{mg})$ was used as an acceptor and dissolved in dehydrate acetonitrile $(5 \mathrm{ml})$, all purchased from Aldrich Co. Inc. The samples were then washed three times in acetonitrile for $30 \mathrm{~s}$, and dried in a vacuum chamber.

The film thickness was measured through scanning force microscope (SFM; CP-II microscope, Veeco Instruments Inc., Plainview, NY, USA). The instrument was equipped with a commercial silicon cantilever with a spring constant and resonant frequency of $\sim 40 \mathrm{~N} \mathrm{~m}^{-1}$ and $300 \mathrm{KHz}$, respectively. The film was first scratched either in manipulation mode by the cantilever or manually, using a narrow needle. The height difference between the resulting groove and the surrounding scratch-free flat area of the film was then measured using the intermittent-contact mode of the SFM.

Transmittance spectra were measured using a ultraviolet-visible spectrometer (V-630, JASCO Co. Ltd., Tokyo, Japan). In the absence of doping, P3HT generally exhibits a strong absorbance peak at $\sim 550 \mathrm{~nm}$; therefore, the transmittance value at this wavelength was used to monitor the transmittance of the films.

The electrical conductivity was measured with a low-resistivity meter (LORESTA GP, Mitsubishi Chemical Co. Ltd., Tokyo, Japan) using a fourpin probe and the contact-current method, accounting for the resistivity correction factor of air at room temperature. Measurements were taken at several different points on the film, and the results for each sample were averaged.

\section{RESULTS AND DISCUSSION}

Figures 1a and c show the SFM images of the cantilever-scratched nanofiber and conventional non-nanofibrillar P3HT films, respectively. The bright vertical band near the center of the images represents the cutting scraps at the scratch edge, which separates the film and groove regions on the left and right, respectively. For the nanofiber film, an entangled nanofiber network was observed in the film area, whereas the surface of the conventional film appeared smooth and without any fine morphology. Figures $1 \mathrm{~b}$ and $\mathrm{d}$ show the corresponding height profiles obtained from the cross sections of the SFM images that were used to determine the film thickness. Figure 2 shows the relationship between the film thickness and the number of drops of suspension or solution used during the spin-casting process. In both the nanofiber and conventional films, an almost linear increase in film thickness with the number of drops used was observed. In addition, both the surface roughness and film thickness increased more rapidly in the nanofiber film because the bulky nature of the stiff nanofibers prevented the formation of a closed-packing film of nanofibers. Incidentally, the thickness of the nanofiber film did not obey the linear relationship when a low number of suspension drops was used. Although a thinner film could be prepared by changing the polymer concentration of the suspension, we did not control the nanofiber film thickness in the thin region because changes to the nanofiber density and connection points between nanofibers affect the conductivity by decreasing polymer concentration.

Figures $3 \mathrm{a}$ and $\mathrm{b}$ show the transmittance spectra of a nanofiber and conventional film, respectively, before and after dipping the films in an $\mathrm{AuCl}_{3}$ solution under identical conditions. As reported previously, ${ }^{20,28}$ the main absorbance peak for the nanofiber film appeared at $520 \mathrm{~nm}$, with narrow shoulders at 550 and $600 \mathrm{~nm}$, which indicated the formation of crystals with face-on stacking of the planar thiophene backbones. In contrast, the main absorbance peak for the conventional film appeared at $550 \mathrm{~nm}$ and had a broad shoulder, which indicated an undeveloped crystal region. In both cases, dipping the films in the $\mathrm{AuCl}_{3}$ solution resulted in decreased absorbance and increased transmittance in the visible region and increased absorbance and decreased transmittance in the nearinfrared region. These results indicate that the nanofiber film was doped as effectively as the conventional film.

Figure 4 shows the time dependence of the transmittance at the peak absorbance wavelength for nanofiber and conventional films doped using $\mathrm{FeCl}_{3}$ and $\mathrm{AuCl}_{3}$, in measurements conducted in air at room temperature. Immediately following the doping process, a
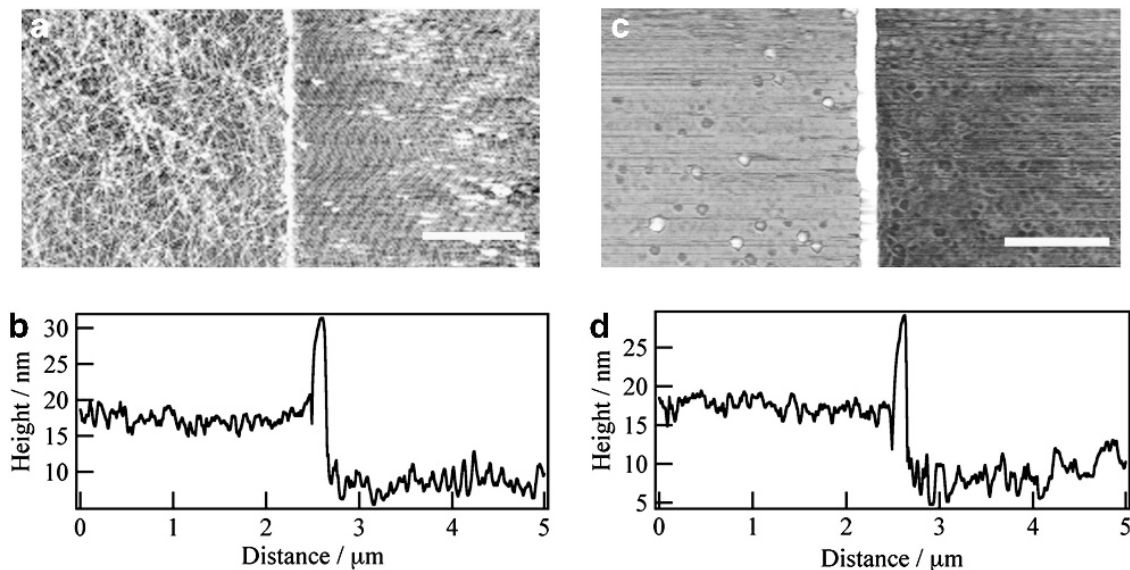

Figure 1 An (a) SFM image and (b) height profile of a nanofiber film scratched by a cantilever, and an (c) SFM image and (d) height profile of a conventional polymer film scratched by a cantilever. The bright vertical band near the center of the images represents the cutting scraps at the scratch edge, separating the film and groove regions on the left and right, respectively. The scale bar represents $2 \mu \mathrm{m}$. 


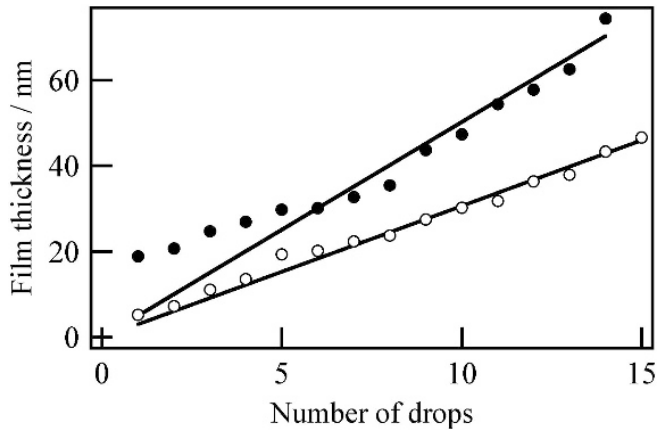

Figure 2 Film thickness as a function of the number of drops of nanofiber suspension (filled circles) and polymer solution (open circles).
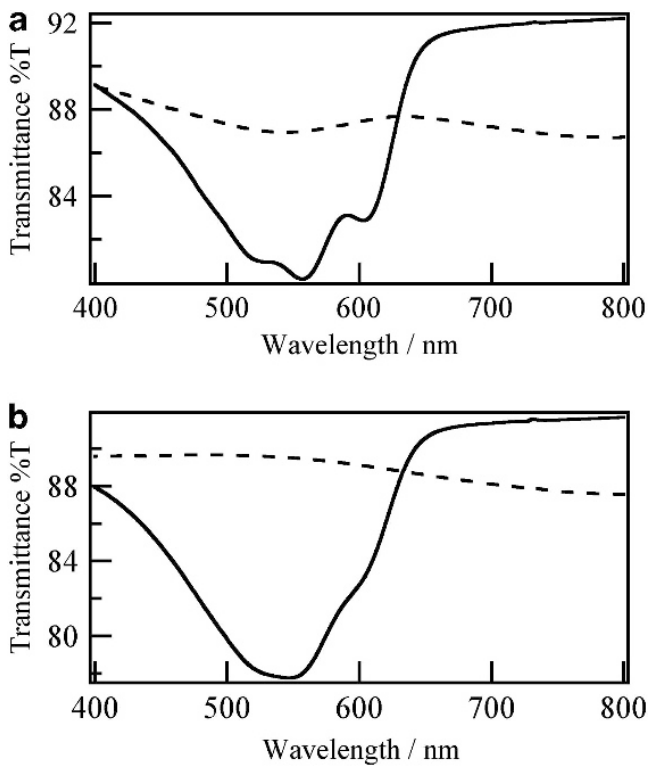

Figure $\mathbf{3}$ Transmittance spectrum of a (a) nanofiber film and (b) conventional polymer film before (solid line) and after doping with $\mathrm{AuCl}_{3}$ (dashed line).

similar doping efficiency was observed for both types of films and dopants. For the nanofiber film, the doping state was comparably stable using either $\mathrm{FeCl}_{3}$ or $\mathrm{AuCl}_{3}$, with the transmittance decreasing only by $\sim 2 \%$ after $72 \mathrm{~h}$. However, the doping state for the conventional film was clearly unstable because the films were thin when kept in air. In particular, for the film doped using $\mathrm{FeCl}_{3}$, the transmittance decreased exponentially and reached the undoped value after $25 \mathrm{~h}$. For the film doped using $\mathrm{AuCl}_{3}$, the doping state also tended to be less stable than that of the nanofiber film.

Figure 5 shows the relationship between the transmittance and the film thickness for nanofiber and conventional films after doping using $\mathrm{AuCl}_{3}$. The transmittance decreased linearly with increasing film thickness. A transmittance of $>85 \%$ was maintained for a film thickness of up to $\sim 50 \mathrm{~nm}$ for the nanofiber film and up to $\sim 30 \mathrm{~nm}$ for the conventional film. Although nanofiber films thinner than $20 \mathrm{~nm}$ were not fabricated, this study found that for thicknesses $>20 \mathrm{~nm}$, the transmittance was higher in nanofiber films than in conventional films doped under identical conditions. It is suggested that two factors contributed to this increase in transmittance. The first
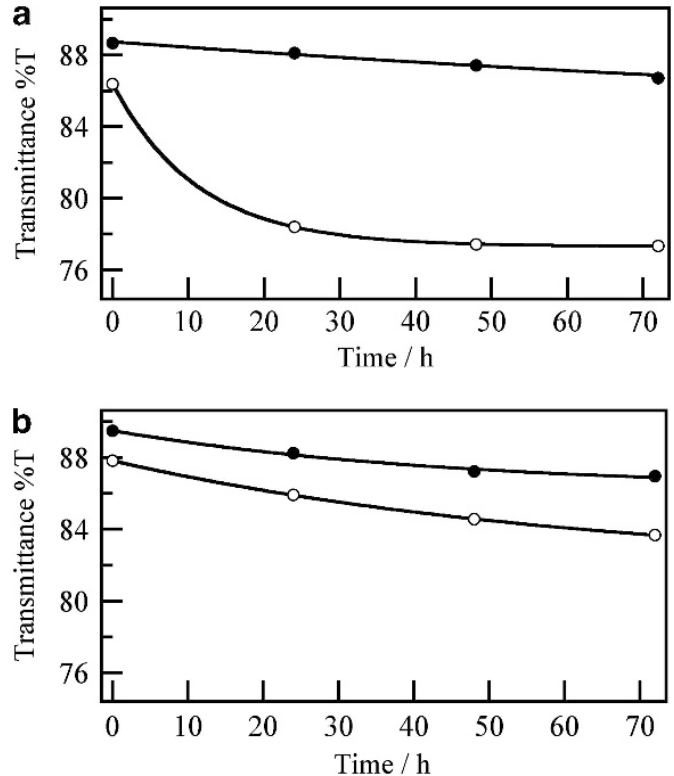

Figure 4 Time dependence of the transmittance at the peak absorbance wavelength for a nanofiber film (filled circles) and a conventional film (open circles), doped using (a) $\mathrm{FeCl}_{3}$ and (b) $\mathrm{AuCl}_{3}$; measurements were carried out in air at room temperature.

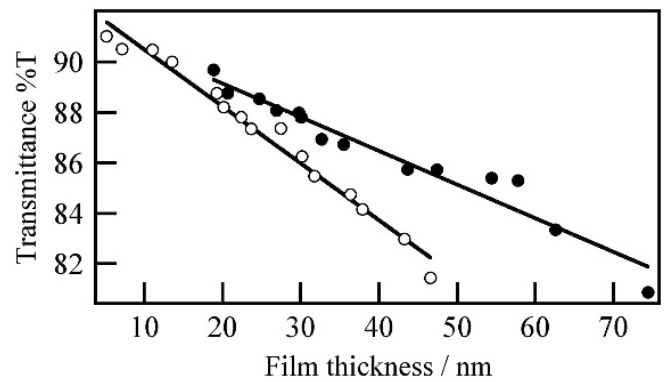

Figure 5 Dependence of transmittance on film thickness for a nanofiber film (filled circles) and conventional film (open circles) after doping with $\mathrm{AuCl}_{3}$.

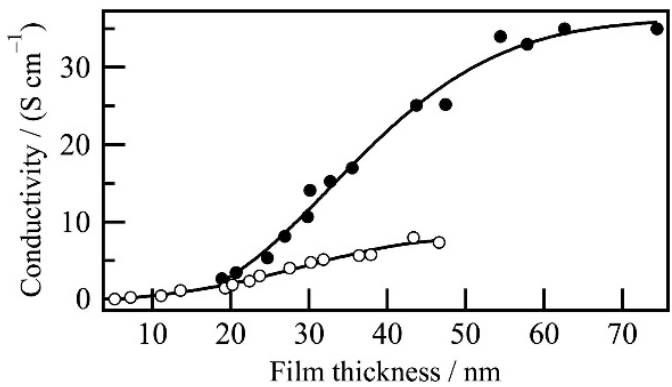

Figure 6 Conductivity of a nanofiber (filled circles) and a conventional film (open circles) as a function of film thickness. 


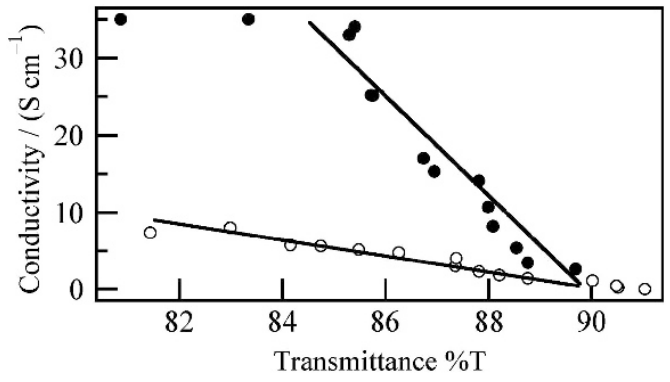

Figure 7 Relationship between transmittance and conductivity for nanofiber films (filled circles) and conventional films (open circles).

is the presence of a large number of voids in the nanofiber films, which are clearly shown in the SFM image in Figure 1a. Although some scattering of light occurs because of the difference in the refractive index between the nanofibers and the voids, the main influence leading to the higher transmittance was the reduced amount of light absorbed by the large number of voids. The second factor is the higher doping concentration that is due to the large surface area offered by the nanofibers during the dipping process. Thus, from the standpoint of transmittance, the results indicate that the nanofiber films offer advantages over conventional films.

Figure 6 shows the conductivity of the nanofiber and conventional films as a function of film thickness. In both types of film, the conductivity increased with thickness and then reached saturation. The use of the four-pin probe and contact-current method should make conductivity independent of the film thickness, but similar tendencies have been commonly reported in the thin film region of various conductors and semiconductors. Various reasons have been suggested for this occurrence, including mobility and crystallinity dependent on the film thickness, surface scattering due to the film thickness comparable to the mean free path of carriers, the discontinuity of the film and the quantum size effect. ${ }^{29,30}$ In particular, inhomogeneous doping, with the formation of partially charged domains, has been suggested as one explanation for the conductivity being affected by film thickness in the conducting polymer. ${ }^{31,32}$ In our study, the effects mentioned above also affect the conductivity. Furthermore, in this study, the conductivity of the nanofiber film was higher than that of the conventional film of the same film thickness fabricated under the same doping conditions. The advantage of the nanofiber film in conductivity is also attributable to following factors. In a previous study, ${ }^{20,21}$ nanofibers with a higher crystallinity were reported to exhibit higher conductivity and carrier mobility. In the present study, higher conductivity is also attributed to the high crystallinity of the nanofiber structure. Another factor is the higher doping concentration in the nanofiber films with the larger surface area, as discussed above. In addition, the effective pathway due to the anisotropic structure of nanofibers may also contribute to the high conductivity. Thus, the conductivity results also indicate that nanofiber films offer advantages over conventional polymer films.

Figure 7 shows the relationship between the transmittance and the conductivity for both nanofiber and conventional films. A tradeoff between the two properties was observed, which is normally the case for general transparent conductive films. It is clear that the nanofiber films offered advantages over the conventional polymer films, with the nanofiber films exhibiting a higher conductivity than the conventional films with the same transmittance; this difference is attributed to the voids created by the bulky network of the nanofibers and the high doping concentration that results from the large surface area. However, compared with the materials used in actual touch panels, the conductivity of a nanofiber film with a transmittance of over $88 \%$ was an order of magnitude less than that required. Further work will be needed to improve the stability of the doping state and the mechanical fragility of the nanofiber films.

\section{SUMMARY}

A comparison of the electrical and optical properties of nanofiber and conventional P3HT films was carried out. The nanofiber films could be doped more efficiently than the conventional films and exhibited a higher conductivity (up to $34 \mathrm{~S} \mathrm{~cm}^{-1}$ ) for the same transmittance ( $\sim 85 \%)$. The advantage of the nanofiber films was due to the presence of effective conducting pathways, a large void fraction in the bulky nanofiber network, and the high doping concentration that arises from the large surface area of the nanofibers.

\section{ACKNOWLEDGEMENTS}

This work was supported in part by a grant-aid for Scientific Research (B) (No. 20350102) from the Ministry of Education, Culture, Sports, Science and Technology of Japan, and an Adaptable and Seamless Technology Transfer Program through Target-Driven R\&D (AS231Z04000D) from the Japan Science and Technology Agency.

1 Zhang, H. \& Li, C. Chemical synthesis of transparent and conducting polyanilinepoly(ethylene terephthalate) composite films. Synth. Met. 44, 143 (1991).

2 Cao, Y., Treacy, G. M., Smith, P. \& Heeger, A. J. Solution-cast films of polyaniline: optical-quality transparent electrodes. Appl. Phys. Lett. 60, 2711 (1992).

3 Cao, Y., Treacy, G. M., Smith, P. \& Heeger, A. J. Optical-quality transparent conductive polyaniline films. Synth. Met. 57, 3526 (1993).

4 Byun, S. W. \& Im, S. S. Degradation kinetics of electrical conductivity in transparent polyaniline-nylon 6 composite films. Synth. Met. 69, 219 (1995).

5 Wan, M. X., Li, M., Li, J. C. \& Liu, Z. X. Transparent and conducting coatings of polyaniline composite. Thin Solid Films 259, 188 (1995).

6 Bayer, A. G. New polythiophene dispersions, their preparation and their use. European Patent 440957 (1991).

7 Gevaert, Agfa. Antistatic plastic parts. European Patent 564911 (1993).

8 Jonas, F., Krafft, W. \& Muys, B. Poly(3, 4-ethylenedioxythiophene): conductive coatings, technical applications and properties. Macromol. Symp. 100, 169 (1995).

9 Groenendaal, L. B., Jonas, F., Freitag, D., Pielartzik, H. \& Reynolds, J. R. Poly (3,4-ethylenedioxythiophene) and its derivatives: Past, present, and future. Adv. Mater. 12,481 (2000).

10 Yan, H., Jo, T. \& Okuzaki, H. Highly conductive and transparent poly(3,4-ethylenedioxythiophene)/poly(4-styrenesulfonate) thin films. Polym. J. 41, 1028 (2009).

11 Apperloo, J. J., van Haare, J. A. E. H. \& Janssen, R. A. J. Transparent highly oxidized conjugated polymer films from solution. Synth. Met. 101, 417 (1999).

12 Park, J. G., Kim, G. T., Kristic, V., Kim, B., Lee, S. H., Roth, S., Burghard, M. \& Park, Y. W. Nanotransport in polyacetylene single fiber: toward the intrinsic properties. Synth. Met. 119, 53 (2001)

13 Aleshin, A. N., Lee, H. J., Park, Y. W. \& Akagi, K. One-dimensional transport in polymer nanofibers. Phys. Rev. Lett. 93, 196601 (2004).

14 Pinto, N. J., Johnson, Jr A. T., MacDiarmid, A. G., Mueller, C. H., Theofylaktos, N., Robinson, D. C. \& Miranda, F. A. Electrospun polyaniline/polyethylene oxide nanofiber field-effect transistor. Appl. Phys. Lett. 83, 4244 (2003).

15 Huang, J. \& Kaner, R. B. A general chemical route to polyaniline nanofibers. J. Am. Chem. Soc. 126, 851 (2004).

16 Wan, M., Wei, Z., Zhang, Z., Zhang, L., Huang, K. \& Yang, Y. Studies on nanostructures of conducting polymers via self-assembly method. Synth. Met. 135-136, 175 (2003).

$17 \mathrm{Ihn}$, K. J., Moulton, J. \& Smith, P. Whiskers of poly (3-alkylthiophene)s. J. Polym. Sci. Part B: Polym. Phys. 31, 735 (1993).

18 Merlo, J. A. \& Frisbie, D. Field effect conductance of conducting polymer nanofibers. J. Polym. Sci. Part B: Polym. Phys. 41, 2674 (2003).

19 Wang, Y. \& Jing, X. Transparent conductive thin films based on polyaniline nanofibers. Mater. Sci. Eng. B 138, 95 (2007).

20 Samitsu, S., Shimomura, T., Heike, S., Hashizume, T. \& Ito, K. Field-effect carrier transport in poly(3-alkylthiophene) nanofiber networks and isolated nanofibers. Macromolecules 43, 7891 (2010).

21 Shimomura, T., Takahashi, T., Ichimura, Y., Nakagawa, S., Noguchi, K., Heike, S. \& Hasuizume, T. Relationship between structural coherence and intrinsic carrier transport in an isolated poly(3-hexylthiophene) nanofiber. Phys. Rev. B 83, 115314 (2011).

22 Merlo, J. A. \& Frisbie, C. D. Field effect transport and trapping in regioregular polythiophene nanofibers. J. Phys. Chem. B 108, 19169 (2004).

23 Chang, J. F., Sun, B. Q., Breiby, D. W., Nielsen, M. M., Solling, T. I., Giles, M., McCulloch, I. \& Sirringhaus, H. Enhanced mobility of poly(3-hexylthiophene) 
transistors by spin-coating from high-boiling-point solvents. Chem. Mater. 16, 4772 (2004).

24 Mas-Torrent, M., den Boer, D., Durkut, M., Hadley, P. \& Schenning, A. P. H. J. Field effect transistors based on poly(3-hexylthiophene) at different length scales. Nanotechnology 15, S265 (2004).

25 Yang, H. C., Shin, T. J., Yang, L., Cho, K., Ryu, C. Y. \& Bao, Z. N. Effect of mesoscale crystalline structure on the field-effect mobility of regioregular poly(3-hexyl thiophene) in thin-film transistors. Adv. Func. Mater. 15, 671 (2005).

26 Kim, D. H., Jang, Y., Park, Y. D. \& Cho, K. Controlled one-dimensional nanostructures in poly(3-hexylthiophene) thin film for high-performance organic field-effect transistors. J. Phys. Chem. B 110, 15763 (2006).

27 Liu, J. H., Arif, M., Zou, J. H., Khondaker, S. I. \& Zhai, L. Controlling poly(3-hexylthiophene) crystal dimension: nanowhiskers and nanoribbons. Macromolecules 42, 9390 (2009).
28 Oosterbaan, W. D., Bolsee, J. C., Gadisa, A., Vrindts, V., Bertho, S., D'Haen, J., Cleij, T. J., Lutsen, L., McNeill, C. R., Thomsen, L., Manca, J. V. \& Vanderzande, D. Alkyl-chain-length-independent hole mobility via morphological control with poly(3alkylthiophene) nanofibers. Adv. Func. Mater. 20, 792 (2010).

29 Minami, T., Sato, H., Nanto, H. \& Takata, S. Group III impurity doped zinc oxide thin films prepared by RF magnetron sputtering. Jpn J. Appl. Phys. 24, L781 (1985).

30 Maissel, L. I. in Handbook of Thin Film Technology (eds Maissel, L. I. \& Glang, R.) Ch. 13 (McGraw-Hill, New York, 1970).

31 Kivelson, S. \& Heeger, A. J. First order phase transition to a metallic state in PA: A strong-coupling polaronic metal. Phys. Rev. Lett. 55, 308 (1985).

32 Kim, J., Sohn, D., Sung, Y. \& Kim, E.-R. Fabrication and characterization of conductive polypyrrole thin film prepared by in situ vapor-phase polymerization. Synth. Met. 132, 309 (2003). 